\title{
Tentativa de suicídio e suicídio no Brasil: análise epidemiológica
}

Daniel Augusto da Silva ${ }^{1}$ (D), João Fernando Marcolan ${ }^{1}$ (D)

\section{RESUMO}

Objetivo: Descrever o perfil epidemiológico sobre tentativas e mortes por suicídio no Brasil. Método: Pesquisa retrospectiva, quantitativa, com dados obtidos em setembro de 2020 no banco de dados do Departamento de Informática do Sistema Único de Saúde do Brasil. Resultados: Aumento gradual no número de notificações de violência autoprovocada de 497,5\% entre 2011 e 2018. Para tentativa de suicídio, em 2018, mulheres foram 68,9\%; entre 20 e 59 anos com 65,6\%; 49,4\% em pessoas brancas. Quanto ao suicídio no período 2009 a 2018, 108.020 óbitos; maiores taxas no sexo masculino; dados de 2018: faixas etárias com distribuição semelhantes; maioria pessoas brancas (49,2\%) e pardas (42,8\%), há importantes diferenças regionais; em 2017 e 2018 maior percentual proporcional em pessoas com 8 a 11 anos de estudo, 33,1 e 35,6\%. Conclusão: Aumento crescente nas taxas de tentativa de suicídio e suicídio, com especificidades por região e estado brasileiros; aumento na morte de indígenas, homens, brancos, solteiros, separados e viúvos, com mais anos de escolaridade, em todas as faixas etárias, morte em casa, método de enforcamento.

Descritores: Saúde mental, Suicídio, Tentativa de suicídio, Epidemiologia, Brasil. 


\section{INTRODUÇÃO}

A ideação suicida, a tentativa de suicídio e a morte por suicídio compõem o comportamento suicida, e compreendem as ações relacionadas a intenção voluntária para o autoextermínio, de modo gradual ${ }^{1}$.

O suicídio produz um impacto sobre as famílias, amigos e comunidades. A respeito do alcance desse impacto, cinco ou seis pessoas próximas podem ser atingidas ${ }^{2}$, contudo, esse impacto pode afetar cerca de 60 pessoas $^{3}$ e até 135 pessoas $^{4}$.

De acordo com a Organização Mundial da Saúde, no ano de 2016, cerca de 804.000 pessoas morreram por suicídio em todo o mundo, a apontar para uma morte a cada 40 segundos e de 10 a 40 tentativas de suicídio para cada morte por suicídio, a depender da região do planeta ${ }^{1,5}$.

No Brasil houve registro de 183.484 mortes por suicídio entre 1996 e 2016, crescimento de 69,6\% nos casos de suicídio neste período ${ }^{6}$. Conforme dados do DATASUS, o Brasil em 2018 registrou 12.733 mortes por suicídio, o que importa na taxa de 6,1 suicídios para cada 100.000 habitantes, a se traduzir em cerca de 35 mortes por dia nesse ano, e manter a posição de oitavo país com mais suicídios no mundo em números absolutos ${ }^{1}$.

Estima-se que uma pessoa a cada 45 minutos morre por suicídio no Brasil, embora não tenhamos dados confiáveis suficientes devido a não termos um sistema de vigilância ao comportamento suicida ainda adequado, apesar das melhorias ocorridas nos últimos anos. Isso faz com que a subnotificação e não notificação ainda sejam muito altas, com mascaramento dos dados como diagnóstico, muitas vezes por solicitação de familiares por vergonha e estigma, a esconder a realidade e nos oferecer taxas baixas?

A subnotificação e o sub-registro são reais e esculpem uma realidade não verdadeira, e, ainda, é importante citar o acidente automobilístico, o afogamento, o envenenamento acidental e a morte por causa indeterminada, que por vezes "escondem" a morte por suicídio ${ }^{8}$.

O Brasil, por ser país de dimensão continental, com população maior que 200 milhões de habitantes, por meio do coeficiente nacional de mortalidade por suicídio facilita a ocultação de significativas variações regionais, justificadas por vários fatores específicos a cada localidade. Estudos mostram que as taxas de suicídio mais elevadas estavam em cidades de pequeno e médio porte populacional ${ }^{9-10}$.
Nesta perspectiva, este estudo tem por objetivo analisar os dados epidemiológicos em relação às tentativas de suicídio (2011-2018) e mortes por suicídio (2009-2018) no Brasil.

\section{MÉTODO}

\section{Tipo do estudo}

Trata-se de estudo descritivo, retrospectivo, de série histórica ${ }^{11}$ (2011-2018 para tentativa de suicídio e 2009-2018 para suicídio), com abordagem quantitativa.

\section{População}

Foi inserida no estudo a totalidade dos casos de tentativa de suicídio registrados no Sistema de Notificações e Agravos em Saúde (SINAN), no período de 2011 a 2018, e de mortes por suicídio registradas no Sistema de Informação de Mortalidade (SIM), no período de 2009 a 2018, ambos disponíveis através do Departamento de Informática do Sistema Único de Saúde do Brasil (DATASUS).

\section{Coleta de dados}

Os dados sobre as tentativas de suicídio e mortes por suicídio foram obtidos no mês de setembro de 2020, por acesso ao banco de dados do DATASUS.

$\mathrm{Na}$ seleção dos dados sobre mortalidade, considerou-se, conforme a 10a Classificação Internacional de Doenças (CID-10), as mortes codificadas com X60-X84 (lesões autoprovocadas intencionalmente) ${ }^{12}$. Dados sobre estimativas populacionais foram obtidos no Instituto Brasileiro de Geografia (IBGE).

As variáveis selecionadas para este estudo são as disponíveis na base de dados: a faixa etária, a cor/raça, a escolaridade, o local de ocorrência, o estado civil, o sexo, a região e Unidade Federativa, e os métodos utilizados para o suicídio.

\section{Análise e tratamento dos dados}

Com o acesso aos dados, os mesmos foram tabulados em planilhas, no software Excel da Microsoft, de modo a permitir a análise dos dados com caráter 
retrospectivo e de série histórica. Além disso, realizouse análise estatística descritiva através do programa Statistical Package for Social Sciences (SPSS), versão 20.0, que proporcionou compreender a frequência absoluta e frequência relativa.

Para os cálculos das taxas de morbidade e taxas de mortalidade foram consideradas populações de 100 mil habitantes.

\section{Aspectos éticos}

Trata-se de pesquisa que utiliza informações de acesso público, em banco de dados, cujas informações são agregadas, embora sem possibilidade de identificação individual. Este estudo faz parte de estudo mais amplo que atendeu a legislação brasileira e foi aprovado pelo Comitê de Ética em Pesquisa sob o parecer n. ${ }^{\circ} 2.314 .347$, no ano de 2017.

\section{RESULTADOS}

\section{A tentativa de suicídio no Brasil (2011-2018)}

Em números absolutos, o Brasil tem apresentado aumento gradual no número de notificações de violência autoprovocada. Entre 2011 e 2018, identificou-se aumento de $497,5 \%$ nos casos notificados. A Tabela 1 apresenta a evolução dos casos de violência autoprovocada notificados no Brasil.

Ao analisar os números absolutos de casos notificados de violência autoprovocada conforme o sexo, no Brasil as mulheres são a maioria, com $68,1 \%$ dos casos em 2017 e 68,9\% dos casos em 2018. A disparidade encontrada ocorre no estado do Amazonas, onde a maioria das notificações de violência autoprovocada são de homens, com 53,4\% em 2018.

Para a idade, considerando o ciclo de vida, a fase jovem, com idade entre 20 e 59 anos, apresenta maior percentual de ocorrências de tentativa de suicídio, com $65,6 \%$ no Brasil, em 2018. Em todas as regiões brasileiras, a tentativa de suicídio é maior nessa fase, com variação de $57,5 \%$ na Região Norte e $66,9 \%$ na Região Sudeste. Ainda, para todos os estados brasileiros, essa é a idade com maior ocorrência, sendo que em todos eles o percentual proporcional é acima dos $50 \%$. Quanto à adolescência, com idade entre 10 e 19 anos, é o segundo maior percentual das ocorrências de tentativa de suicídio, com $29,8 \%$ no Brasil, e variação 28,1 a $38,3 \%$ entre as regiões brasileiras.

Para a cor de pele, em 2018 no Brasil, 49,4\% das notificações de violência autoprovocada foram de pessoas brancas, seguido de pessoas pardas com $34,2 \%$. Contudo, há de se considerar as diferenças regionais que caracterizam a população brasileira, de forma que em Santa Catarina, o percentual proporcional para pessoas brancas é de $87,6 \%$, e no Amazonas é de $3,8 \%$. Para pessoas pardas, o percentual proporcional varia de $82,7 \%$ em Roraima a 6,3\% em Santa Catarina.

\section{suicídio no Brasil (2009-2018)}

Nos últimos 10 anos (2009 a 2018), dados mais recentes disponibilizados pelo DATASUS, o Brasil registrou, em caráter ascendente, 108.020 óbitos por suicídio, denominadas lesões autoprovocadas voluntariamente. Destes, 38,3\% foram registrados na Região Sudeste, fato que, em números absolutos, se relaciona ao maior quantitativo da população brasileira nessa região.

A Tabela 2 apresenta os dados dos óbitos por suicídio no período de 2009 a 2018 no Brasil, nas Regiões brasileiras e nas Unidades da Federação.

A Figura 1 representa a evolução da Taxa de Mortalidade por suicídios no Brasil, calculada através de dados obtidos do Departamento de Informática do Sistema Único de Saúde (DATASUS) sobre o número de óbitos por suicídio no Brasil no período de 2009 a 2018 e do Instituto Brasileiro de Geografia e Estatística (IBGE), sobre a população residente no país no mesmo período citado acima. As taxas estão calculadas por 100.000 habitantes.

Ao analisar os óbitos por suicídio entre os sexos, no ano de 2018, os cinco maiores percentuais proporcionais de óbitos de homens foram na Bahia $(85,3 \%)$, no Rio Grande do Norte $(84,2 \%)$, no Ceará $(82,3 \%)$, no Paraná $(81,5 \%)$ e no Pará $(80,0 \%)$. 0 estado do Amazonas foi único a apresentar maioria masculina para tentativas de suicídio, provavelmente por ter concentração de população indígena e a ocorrência de mortes por suicídio nesses povos, em sua maioria homens.

Para as mulheres, os cinco maiores percentuais proporcionais de óbitos foram em Roraima (32,4\%), no Amapá $(32,3 \%)$, no Acre $(28,8 \%)$, em Rondônia $(27,2 \%)$ e no Piauí $(26,6 \%)$. 
Tabela 1. Números absolutos de violência autoprovocada notificada no Brasil, Regiões brasileiras e Unidades da Federação, no período de 2011 a 2018. Assis, SP, Brasil, 2020.

\begin{tabular}{|c|c|c|c|c|c|c|c|c|}
\hline Região/Unidade da Federação & 2011 & 2012 & 2013 & 2014 & 2015 & 2016 & 2017 & 2018 \\
\hline Região Norte & 636 & 722 & 1081 & 1112 & 1577 & 1935 & 2819 & 3114 \\
\hline .. Rondônia & 36 & 21 & 43 & 37 & 108 & 180 & 375 & 431 \\
\hline .. Acre & 33 & 74 & 65 & 85 & 243 & 314 & 614 & 515 \\
\hline .. Amazonas & 104 & 88 & 152 & 207 & 416 & 319 & 281 & 341 \\
\hline .. Roraima & 56 & 128 & 166 & 142 & 115 & 149 & 269 & 324 \\
\hline .. Pará & 90 & 69 & 104 & 158 & 177 & 212 & 298 & 254 \\
\hline .. Amapá & 57 & 71 & 104 & 45 & 38 & 61 & 81 & 108 \\
\hline .. Tocantins & 260 & 271 & 447 & 438 & 480 & 700 & 901 & 1141 \\
\hline Região Nordeste & 1995 & 2564 & 3646 & 3638 & 4976 & 5555 & 8623 & 12105 \\
\hline .. Maranhão & 61 & 166 & 173 & 185 & 375 & 198 & 283 & 454 \\
\hline .. Piauí & 198 & 318 & 371 & 360 & 606 & 1045 & 1053 & 1247 \\
\hline .. Ceará & 72 & 109 & 234 & 285 & 661 & 886 & 1400 & 1957 \\
\hline .. Rio Grande do Norte & 107 & 126 & 251 & 233 & 334 & 441 & 728 & 1065 \\
\hline .. Paraíba & 191 & 241 & 186 & 196 & 438 & 285 & 648 & 793 \\
\hline .. Pernambuco & 421 & 443 & 998 & 1076 & 1124 & 1242 & 2169 & 3199 \\
\hline .. Alagoas & 764 & 853 & 972 & 846 & 851 & 804 & 1227 & 1663 \\
\hline .. Sergipe & 5 & 13 & 47 & 42 & 43 & 41 & 99 & 238 \\
\hline .. Bahia & 176 & 295 & 414 & 415 & 544 & 613 & 1016 & 1489 \\
\hline Região Sudeste & 7455 & 10836 & 12422 & 15384 & 20182 & 23301 & 33624 & 43096 \\
\hline .. Minas Gerais & 2377 & 4315 & 5782 & 7454 & 9153 & 8674 & 11273 & 13348 \\
\hline .. Espírito Santo & 68 & 225 & 371 & 867 & 1238 & 1580 & 2001 & 3240 \\
\hline .. Rio de Janeiro & 466 & 732 & 859 & 1121 & 1656 & 2125 & 3570 & 4246 \\
\hline .. São Paulo & 4544 & 5564 & 5410 & 5942 & 8135 & 10922 & 16780 & 22262 \\
\hline Região Sul & 3557 & 5342 & 6403 & 7524 & 10142 & 11562 & 18766 & 24264 \\
\hline .. Paraná & 581 & 1367 & 1741 & 2356 & 3892 & 4754 & 7777 & 9950 \\
\hline .. Santa Catarina & 1075 & 1480 & 2220 & 2476 & 2948 & 3131 & 4470 & 5816 \\
\hline .. Rio Grande do Sul & 1901 & 2495 & 2442 & 2692 & 3302 & 3677 & 6519 & 8498 \\
\hline Região Centro-Oeste & 1297 & 1700 & 1918 & 2050 & 2812 & 3136 & 4369 & 6693 \\
\hline .. Mato Grosso do Sul & 905 & 886 & 1018 & 1074 & 1244 & 1300 & 1734 & 1928 \\
\hline .. Mato Grosso & 86 & 209 & 178 & 225 & 235 & 273 & 477 & 641 \\
\hline .. Goiás & 218 & 472 & 561 & 580 & 977 & 1051 & 1395 & 2234 \\
\hline .. Distrito Federal & 88 & 133 & 161 & 171 & 356 & 512 & 763 & 1890 \\
\hline Brasil & 14940 & 21164 & 25470 & 29708 & 39689 & 45489 & 68201 & 89272 \\
\hline
\end{tabular}

Fonte: Elaborado pelos autores com dados do DATASUS, 2020.

A análise relacionada às idades revelou que a faixa etária com maior proporção de óbitos em 2018 no Brasil foi de pessoas com idade entre 30 e 39 anos $(20,7 \%)$, seguido de pessoas com idade entre 20 e 29 anos $(19,7 \%)$ e idade entre 40 e 49 anos $(18,1 \%)$. O suicídio de idosos, pessoas com 60 anos e mais, correspondeu a $17,9 \%$ das ocorrências no mesmo ano. Na série histórica de 1996 a 2018 as taxas aumentaram
$162,2 \%$ na faixa etária de 60 a 69 anos, $141,4 \%$ entre 70 e 79 anos e $189,3 \%$ com 80 anos e mais.

Sobre a cor de pele, o Brasil registra a maioria de óbitos por suicídio em pessoas com cor de pele branca $(49,2 \%)$ e parda $(42,8 \%)$, todavia, na análise das Regiões brasileiras e Unidades da Federação são perceptíveis as diferenças regionais que são características do Brasil. Nas regiões brasileiras, 
Tabela 2. Óbitos por suicídio no Brasil, Regiões brasileiras e Unidades da Federação, no período de 2009 a 2018. Assis, SP, Brasil, 2020.

\begin{tabular}{|c|c|c|c|c|c|c|c|c|c|c|}
\hline Região/Unidade da Federação & 2009 & 2010 & 2011 & 2012 & 2013 & 2014 & 2015 & 2016 & 2017 & 2018 \\
\hline Região Norte & 593 & 624 & 692 & 694 & 759 & 708 & 881 & 826 & 896 & 991 \\
\hline .. Rondônia & 85 & 82 & 78 & 73 & 86 & 84 & 109 & 103 & 113 & 125 \\
\hline .. Acre & 31 & 41 & 41 & 43 & 44 & 49 & 39 & 56 & 64 & 59 \\
\hline .. Amazonas & 152 & 162 & 188 & 185 & 225 & 233 & 263 & 194 & 207 & 234 \\
\hline .. Roraima & 32 & 34 & 34 & 38 & 33 & 15 & 52 & 59 & 50 & 37 \\
\hline .. Pará & 188 & 188 & 222 & 240 & 232 & 208 & 266 & 277 & 301 & 350 \\
\hline .. Amapá & 26 & 30 & 37 & 21 & 45 & 34 & 53 & 36 & 46 & 62 \\
\hline .. Tocantins & 79 & 87 & 92 & 94 & 94 & 85 & 99 & 101 & 115 & 124 \\
\hline Região Nordeste & 2101 & 2123 & 2297 & 2336 & 2494 & 2393 & 2540 & 2722 & 2981 & 2996 \\
\hline .. Maranhão & 156 & 208 & 218 & 206 & 242 & 255 & 280 & 294 & 318 & 313 \\
\hline .. Piauí & 207 & 201 & 234 & 233 & 227 & 244 & 271 & 321 & 317 & 331 \\
\hline .. Ceará & 501 & 488 & 553 & 510 & 590 & 566 & 565 & 590 & 644 & 655 \\
\hline .. Rio Grande do Norte & 144 & 137 & 177 & 171 & 157 & 169 & 156 & 181 & 180 & 196 \\
\hline .. Paraíba & 166 & 158 & 163 & 189 & 199 & 158 & 221 & 181 & 250 & 237 \\
\hline .. Pernambuco & 328 & 285 & 291 & 332 & 320 & 325 & 308 & 396 & 438 & 430 \\
\hline .. Alagoas & 111 & 85 & 104 & 109 & 143 & 118 & 116 & 112 & 104 & 137 \\
\hline .. Sergipe & 111 & 129 & 125 & 109 & 125 & 110 & 120 & 115 & 127 & 134 \\
\hline .. Bahia & 377 & 432 & 432 & 477 & 491 & 448 & 503 & 532 & 603 & 563 \\
\hline Região Sudeste & 3570 & 3735 & 3900 & 4002 & 3959 & 4283 & 4323 & 4249 & 4635 & 4675 \\
\hline .. Minas Gerais & 1123 & 1102 & 1258 & 1264 & 1159 & 1357 & 1303 & 1302 & 1515 & 1530 \\
\hline .. Espírito Santo & 150 & 160 & 162 & 178 & 158 & 172 & 189 & 175 & 207 & 239 \\
\hline .. Rio de Janeiro & 321 & 509 & 433 & 463 & 437 & 522 & 531 & 573 & 607 & 699 \\
\hline .. São Paulo & 1976 & 1964 & 2047 & 2097 & 2205 & 2232 & 2300 & 2199 & 2306 & 2207 \\
\hline Região Sul & 2279 & 2154 & 2156 & 2357 & 2365 & 2319 & 2494 & 2602 & 2862 & 2891 \\
\hline .. Paraná & 648 & 588 & 593 & 629 & 655 & 620 & 716 & 760 & 774 & 915 \\
\hline .. Santa Catarina & 519 & 530 & 520 & 548 & 568 & 587 & 637 & 674 & 739 & 735 \\
\hline .. Rio Grande do Sul & 1112 & 1036 & 1043 & 1180 & 1142 & 1112 & 1141 & 1168 & 1349 & 1241 \\
\hline Região Centro-Oeste & 831 & 812 & 807 & 932 & 956 & 950 & 940 & 1034 & 1121 & 1180 \\
\hline .. Mato Grosso do Sul & 205 & 188 & 211 & 210 & 228 & 204 & 230 & 223 & 259 & 268 \\
\hline .. Mato Grosso & 190 & 161 & 158 & 185 & 177 & 157 & 145 & 178 & 197 & 226 \\
\hline .. Goiás & 307 & 315 & 338 & 402 & 427 & 454 & 435 & 481 & 497 & 499 \\
\hline .. Distrito Federal & 129 & 148 & 100 & 135 & 124 & 135 & 130 & 152 & 168 & 187 \\
\hline Brasil & 9374 & 9448 & 9852 & 10321 & 10533 & 10653 & 11178 & 11433 & 12495 & 12733 \\
\hline
\end{tabular}

Fonte: Elaborado pelos autores com dados do DATASUS, 2020.

os percentuais proporcionais de óbito por suicídio relacionado a cor de pele branca e parda são, respectivamente, 12,2 e $75,0 \%$ na Região Norte, 15,2 e $75,7 \%$ na Região Nordeste, 58,7 e 33,2\% na Região Sudeste, 87,0 e 8,9\% na Região Sul e 36,0 e $53,9 \%$ na Região Centro-Oeste.

Destaca-se o aumento de $176 \%$ de suicídios entre os indígenas, considerado o período de 2000 a 2018.
Entre os estados brasileiros, os percentuais extremos dos óbitos de pessoas brancas vão de $90,9 \%$ no Rio Grande do Sul para 2,9\% em Alagoas. Do mesmo modo, pessoas pardas são $89,6 \%$ dos óbitos em Sergipe e 4,1\% no Rio Grande do Sul.

Para a escolaridade, no Brasil, excluindo as informações ignoradas, em 2017 e 2018 o maior percentual proporcional foi de pessoas com 8 a 11 


\section{Suldele mortality rate in Bracil}

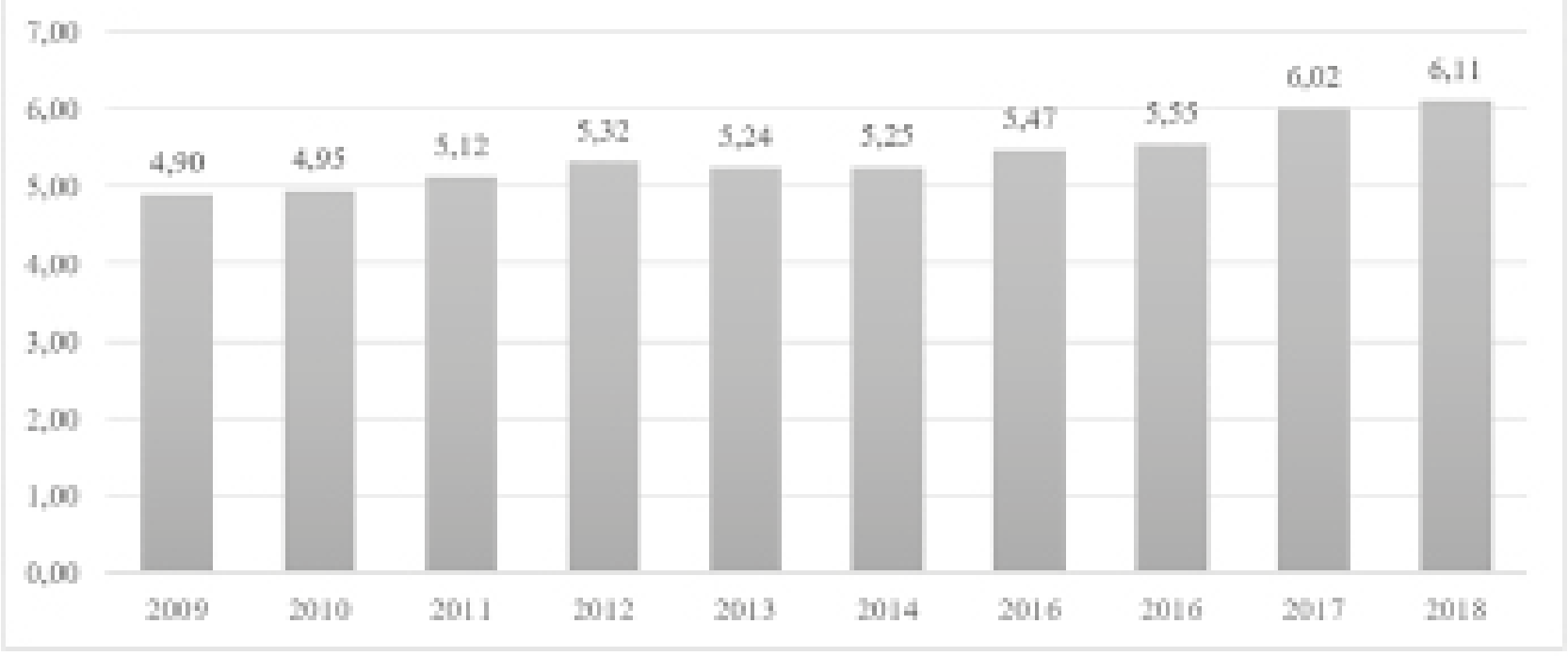

Figura 1. Evolução da Taxa de Mortalidade por suicídios no Brasil de 2009 a 2018. Assis, SP, Brasil, 2020.

Fonte: Elaborado pelos autores com dados do DATASUS, 2020.

anos de estudo, $33,1 \%$ e $35,6 \%$, respectivamente. Nos anos anteriores, 2013, 2014 e 2015, o maior percentual era de pessoas com 4 a 7 anos de estudo, $33,5,32,9$ e $33,6 \%$, respectivamente. Na série histórica de 1996 a 2018 as taxas aumentaram 824\% para as pessoas entre 8 e 11 anos de escolaridade e $520,3 \%$ para as com 12 anos e mais de estudo.

Para o estado civil, pessoas solteiras são a maioria das ocorrências no Brasil e em todas as regiões e estados brasileiros, mesmo com diferenças de $87,1 \%$ no Amapá e $31,2 \%$ na Paraíba.

A maioria dos indivíduos tentaram suicídio e se suicidaram em casa; o método mais utilizado e em ascensão foi enforcamento, seguido por métodos em descenso como intoxicação exógena e arma de fogo, respectivamente.

De modo geral, todas as regiões tiveram aumento nas taxas de suicídio na série histórica de 1996 a 2018, o Norte com 81,1\%, Nordeste com $126,5 \%$, Sudeste com $26,6 \%$, Sul com $17,8 \%$ e Centro-Oeste com 28,5\%. Quanto aos estados com maiores aumentos para taxas de suicídio, temos Piauí $(464,6 \%)$, Paraíba $(402,6 \%)$, Maranhão $(355,6 \%)$ todos no Nordeste, Tocantins $(264,1 \%)$ no Centro-Oeste e Acre $(227,9 \%)$ no Norte. Ainda, historicamente, os estados da Região Sul têm taxas mais elevadas quando comparadas às taxas calculadas nos demais estados brasileiros.

\section{DISCUSSÃO}

A tentativa de suicídio é um dos principais indicadores do risco de suicídio, entretanto, a subnotificação resulta no fornecimento de informações atenuadas ${ }^{1,13-14}$. É estimado que ao menos 20 tentativas de suicídio ocorram para cada morte por suicídio de adulto, o que é traduzido em uma tentativa de suicídio a cada segundo ${ }^{7}$. Os dados brasileiros passam a ser melhor coletados e notificados a partir de 2014, devido à legislação em vigor, e isso influenciou sobremaneira para o aumento elevado de casos notificados de 2011 a $2018(497,5 \%)$.

No Brasil, o boletim com perfil epidemiológico com informações sobre tentativas de suicídio e suicídio por intoxicação exógena no período de 2007 a 2017, revelou 220.045 notificações de intoxicação exógena caracterizada como tentativa de suicídio, e destas, $69,9 \%$ foram registradas em pessoas do sexo feminino ${ }^{15}$.

Em Minas Gerais, na cidade de Uberaba, estudo que utilizou o banco de dados do Sistema Nacional de Notificação (SINAN), com informações das fichas de notificação respectivas à violência e à intoxicação exógena ocorridas em 2014, revelou 89 casos de tentativas de suicídio por intoxicação exógena, dos quais $68(76,4 \%)$ eram mulheres ${ }^{16}$. 
Na cidade de Palmas (TO), das 656 notificações de tentativa de suicídio no período de 2010 a 2014, $67,1 \%$ foram de mulheres ${ }^{17}$.

Quanto ao suicídio no Brasil, chama atenção o fato da ascensão constante no período de 2013 a 2018, que coincide com melhor notificação de casos, a distribuição sem grandes diferenças estatísticas entre as faixas etárias e o aumento também constante entre os indivíduos com maior tempo de estudo.

Os dados sobre dar fim a própria vida pelo suicídio, conforme Estimativas Globais de Saúde da Organização Mundial da Saúde para o ano de 2016 revelou a taxa de mortalidade por suicídio maior nos homens $(13,7$ por 100.000 habitantes) do que nas mulheres $(7,5 \text { por } 100.000 \text { habitantes })^{18}$.

Ensaio sobre o suicídio feminino, aponta a tentativa por parte da psicologia e da psiquiatria para justificar este comportamento, que seria resultado de situações inerentes ao gênero feminino, ou feminilidade, como o desejo de chamar a atenção para si, traços histéricos, variações hormonais, volatilidade de conduta, impulsividade, emotividade excessiva, falta de racionalidade e pragmatismo. No entanto, as autoras propõem uma reflexão quanto aos aspectos culturais e de gênero, em amplitude histórica ${ }^{19}$.

Na compreensão do porquê de as mulheres terem mais tentativas de suicídio do que os homens, alguns fatores são apontados e correlacionam essa vulnerabilidade, como a construção social do gênero; a maior prevalência de depressão; a maior ocorrência de distúrbios alimentares; problemas com a imagem corporal; a gravidez indesejada; a psicose pósparto; a grande ocorrência de ideação suicida após aborto induzido e nas situações de baixos níveis de estrogênio e serotonina; a grande vulnerabilidade à perda de filhos; a violência doméstica contra elas e os filhos e o abuso sexual ${ }^{20-21}$.

O Estudo Global de Cargas de Doenças, realizado em 2015, revelou estar o suicídio, na faixa etária de 10 a 24 anos entre as cinco principais causas de mortalidade em todas as regiões, exceto na África ${ }^{22-23}$. É grande a vulnerabilidade de adolescentes e jovens adultos ao comportamento suicida. No mundo, quase um terço de todos os suicídios é de pessoas jovens ${ }^{24}$.

É uma situação de saúde pública preocupante e complexa, por envolver série de fatores de risco, que, nesta idade, podem ser citados os transtornos mentais, como os de humor, uso de álcool e outras drogas e transtornos alimentares; fatores psicológicos, como baixa autoestima, desesperança e impulsividade; os fatores psicossociais da adversidade, como exposição a violências, ambiente de conflito, exposição a eventos estressantes traumáticos como abuso ou vitimização, baixa confiança e baixa comunicação com mãe e pai, lar desestruturado e pequeno número de amigos, migração, pobreza. Destaca-se a existência de histórico de violência, brigas e agressões, transgressão às leis e problemas de conduta em geral em adolescentes com comportamento suicida ${ }^{24-27}$.

Neste contexto de análise do comportamento suicida por ciclos vitais, a velhice se destaca pela presença de comportamento suicida nesta população e pela população mundial estar envelhecendo, a culminar em boa parte da população nesta faixa etária.

O envelhecimento é um processo inerente ao ciclo de vida. A este processo, há alterações identificadas que se referem aos aspectos físicos, financeiros, psicológicos, emocionais e estruturais. Nesta etapa, carregada de processos de mudança mútua em diferentes perspectivas, o idoso tem a necessidade de apoio para adaptação saudável de sua vida, de modo que, quando não acontece, a falta de expectativa de vida futura o torna vulnerável ao suicídio ${ }^{28}$.

A este processo de envelhecimento, vale a observação do significado ao qual este exerce às pessoas, de forma que pode ser influenciado pelo contexto vivido. No Brasil, o envelhecimento assume característica de inatividade, de forma que o indivíduo alcança uma época que deixa de realizar muitas das atividades que outrora eram de sua competência ${ }^{29}$.

A esta situação, atrelam-se expressivas perdas emocionais: o contato com as pessoas, o trabalho, a contribuição econômica para o lar, a desvalorização do senso de pertencimento e utilidade, situações que abrigam potencial transformador negativo aos idosos, que passam a adquirir a autopercepção estigmatizada de inutilidade. Além dessas relações fragilizadas, que geram violências, outros fatores devem ser considerados, como os problemas físicos e incapacitantes como as doenças crônicas, declino funcional; problemas psiquiátricos como depressão, abuso de substâncias lícitas e ilícitas, transtornos de personalidade, comportamentos autodestrutivos, comprometimento cognitivo; problemas psicológicos como sofrimento persistente ou traumático, sentimento de solidão, desesperança e tédio, fragilidade; problemas sociais como sofrer isolamento social, viver em conflitos familiares, 
nível de escolaridade baixo, ter vivenciado mortes e perdas de parentes próximos ou amigos, ausência de religiosidade, inflexibilidade e rigidez em relação a mudanças, particularmente as sociais; problemas econômicos como a falta de autonomia para gerenciar o próprio dinheiro, ausência de seguridade e assistência social; entre outros ${ }^{29-31}$.

Para compreender a relação entre o comportamento suicida e a cor de pele, deve-se considerar o contexto de cada localidade, pois são estes que determinam quais grupos podem ser mais vulneráveis ${ }^{24}$. Ressalte-se que os determinantes sociais do comportamento suicida possuem relação com a violência estrutural, que por sua vez, é associada ao colonialismo, período histórico no qual grupos eram explorados, discriminados, marginalizados e excluídos. São essas pessoas, classificadas nesses grupos, que hoje são marcadas como vulneráveis ${ }^{32}$.

Análise do histórico de notificação para tentativa de suicídio por intoxicação exógena no Brasil no período de 2007 a 2017, revelou a maioria com cor de pele branca $(46,8 \%$ para mulheres e $43,7 \%$ para homens), seguido de cor de pele negra, somadas as cores preta e parda (33,1\% para mulheres e $36,5 \%$ para homens). A informação ignorada teve significativa ocorrência (19,4\% para mulheres e $19,0 \%$ para homens) ${ }^{15}$.

Dados inversos estão em estudo brasileiro que envolveu 86 serviços de urgência e emergência do Sistema Único de Saúde (SUS), localizados em 24 capitais e no Distrito Federal, com registro de 477 ocorrências de violência autoprovocada. Dentre elas, $62,4 \%$ foram de pessoas com cor de pele preta ou parda, e $34,3 \%$ de pessoas com cor de pele branca ${ }^{33}$.

O coeficiente de mortalidade por suicídio na adolescência segundo a raça-cor da pele no Brasil de 2000 para 2010, com dados do DATASUS, os adolescentes de cor de pele preta, parda e indígena foram os mais acometidos pelo suicídio ${ }^{34}$.

No Brasil, no período de 2011 a 2015, 60,4\% dos óbitos por suicídio foram de pessoas solteiras, viúvas ou divorciadas e $31,5 \%$ estavam casados ou em união estável. O percentual de homens e mulheres solteiros também foi maior em estudo com pessoas que cometeram o suicídio, com homens solteiros em $57,9 \%$ e mulheres solteiras, em $40,0 \%$. Quando separados, encontrou $20,0 \%$ das mulheres e $5,2 \%$ dos homens ${ }^{35}$.

Tanto para tentativa de suicídio como suicídio no Brasil deve-se levar em consideração as diferenças regionais com o sul tendo maior população de cor branca, menor taxa de miscigenação, valores socioculturais, religiosidade, mais anos de estudo, parcela pequena de indígenas, muito em função da colonização europeia. Por outro lado, o sudeste e nordeste, com maior miscigenação de raças e povos, diversidade cultural e religiosa, influência de africanos, europeus, indígenas e asiáticos, e o centro-oeste com seu corredor agroindustrial a receber pessoas de todo lugar brasileiro, a expandir e expulsar os indígenas e o norte com suas características da imensidão amazônica e a cultura indígena. Faz-se necessário prestar atenção a esses fatores quando da análise locorregional para comportamento suicida e as variáveis como cor da pele, estado civil, faixa etária, anos de estudo, renda individual e familiar entre outras.

A considerar na discrepância do aumento das taxas de suicídio entre as regiões brasileiras o fato de algumas regiões/estados terem sistema de registros históricos mais confiáveis ao longo dos anos e outras não tinham, com início relativamente recente destes registros.

Este estudo contribui no avanço das práticas de saúde, pois se dedica a analisar o comportamento suicida e a necessidade de investir em ações de promoção à saúde mental e prevenção da morte por suicídio, tendo em vista o diagnóstico situacional exposto pelos casos notificados e registrados nos bancos de dados brasileiros. Colabora para a elaboração e implantação de políticas públicas na área.

As limitações deste estudo se relacionam à utilização de dados secundários, que são conhecidamente passíveis de subnotificações e falhas nos registros.

\section{CONCLUSÃO}

Há aumento significativo e ainda crescente nas taxas de tentativa de suicídio e suicídio no Brasil, com especificidades para cada região e estado brasileiros, com destaque para aumento na morte de indígenas, brancos, com mais anos de escolaridade e melhor coleta e notificação dos dados, embora ainda não seja um sistema de excelência.

\section{REFERÊNCIAS}

1. World Health Organization. Preventing suicide: a global imperative. Geneva: WHO; 2014.

2. Franck MC, Monteiro MG, Limberger RP. Mortalidade por suicídio no Rio Grande do Sul: uma análise transversal 
dos casos de 2017 e 2018. Epidemiologia e Serviços de Saúde. 2020;29(2):e2019512.

3. Penso MA, Sena DPA. A desesperança do jovem e o suicídio como solução. Sociedade e Estado. 2020;35(1):61-81.

4. Cerel J, Brown MM, Maple M, Singleton M, van de Venne J, Moore M, Flaherty C. How many people are exposed to suicide? Not six. Suicide and Life-Threatening Behavior. 2019;49:529-534.

5. World Health Organization. World health statistics 2019: monitoring health for the SDGs, sustainable development goals. Geneva: WHO; 2019

6. Marcolan JF, Silva DA. O comportamento suicida na realidade brasileira: aspectos epidemiológicos e da política de prevenção. Revista M. 2019;4(7):31-44.

7. Marcolan JF. For a public policy of surveillance of suicidal behavior. Rev. Bras. Enferm. [Internet]. 2018;71(Suppl 5):2343-7.

8. Silva DA, Marcolan JF. Epidemiologia do suicídio no Brasil entre 1996 e 2016 e a política pública. RSD [Internet]. 2020;9(2):e79922080

9. Marín-León L, Oliveira HB, Botega NJ. Suicide in Brazil, 2004-2010: the importance of small counties. Rev Panam Salud Publica. 2012;32(5):351-9.

10. Minayo MCS, Pinto LW, Assis SG, Cavalcante FG, Mangas RMN. Trends in suicide mortality among Brazilian adults and elderly, 1980 - 2006. Rev. Saúde Pública [Internet]. 2012;46(2):300-9.

11. Marconi MA, Lakatos EM. Fundamentos da metodologia científica. São Paulo: Atlas; 2021.

12. Organização Mundial da Saúde. CID-10 - Classificação Estatística Internacional de Doenças e Problemas Relacionados à Saúde. São Paulo: Edusp; 2017.

13. Organization for Economic Co-operation and Development. Society at a Glance 2014: OECD Social Indicators; 2014.

14. Stinson JD, Gonsalvez V. Suicide Attempts and SelfHarm Behaviors in Psychiatric Sex Offenders. Sex Abuse. 2013;26(3):252-70.

15. Brasil. Ministério da Saúde. Boletim epidemiológico. n. 24. 2019.

16. Ribeiro NM, Castro SS, Scatena LM, Haas LM. Análise da tendência temporal do suicídio e de sistemas de informações em saúde em relação às tentativas de suicídio. Texto Contexto Enferm, 2018;27(2):e2110016.

17. Fernandes DAA, Ferreira NS, Castro JGD. Perfil epidemiológico das tentativas de suicídio em Palmas-Tocantins, de 2010 a 2014. Tempus, actas de saúde colet. 2016;10(4):9-23.

18. World Health Organization. Suicide in the world: Global Health Estimates. Geneva: WHO; 2019.

19. Marquetti FR, Marquetti FC. Suicídio e feminilidades. Cadernos Pagu. 2017;49:e174921.

20. Correia CM, Gomes NP, Diniz NMF, Andrade ICS, Romano CMC, Rodrigues GRS. Child and adolescente violence: oral story of women who attempted suicide. Rev bras enferm. 2019;72(6):1450-6.
21. Ferreira ACZ, Capistrano FC, Maftum GJ, Kalinke, LP, Maftum MA. Comportamento suicida em pessoas com transtornos relacionados a substâncias. Rev enferm UFPE on line. 2019;13:e241446.

22. GBD 2016 Causes of Death Collaborators, Naghavi M, Abajobir AA, Abbafati C, et al.Global, regional, and national age-sex specific mortality for 264 causes of death, 19802016: a systematic analysis for the Global Burden of Disease Study 2016. Lancet. 2017;390:1151-210.

23. Naghavi M. Global, regional, and national burden of suicide mortality 1990 to 2016: systematic analysis for the Global Burden of Disease Study 2016. BMJ. 2019;364:194.

24. World Health Organization. Preventing suicide: a community engagement toolkit. Geneva: WHO; 2018.

25. Soto-Sanz V, Piqueras JA, Rodríguez-Marín J, Pérez-Vázquez MT, Rodríguez-Jiménez T, Castellví $P$ et al. Self-esteem and suicidal behaviour in youth: A meta-analysis of longitudinal studies. Psicothema (Oviedo). 2019;31(3):246-54.

26. Suárez Colorado $Y$, Campo-Arias A. Asociación entre apego y riesgo suicida en adolescentes escolarizados de Colombia. Rev. chil. pediatr. 2019;90(4):392-8.

27. Gomes CFM, Silva DA. Aspectos epidemiológicos do comportamento suicida em estudantes universitários. RSD [Internet]. 2020;9(5):e38953106.

28. Armond JE, Armond RE, Pereira TC, Chinaia C, Vendramini TL, Rodrigues CL. Self-injury and suicide attempt among the elderly population in the city of São Paulo. J. bras. psiquiatr. 2017;66(2):83-8.

29. Fernandes-Eloi J, Lourenço JRC. Suicídio na Velhice Um estudo de revisão integrativa da literatura. CES Psicol [online]. 2019;12(1):80-95.

30. Salazar JAA, Sapuy LPL, Romero AR, Ramírez JAC. Vulnerabilidad biopsicosocial y suicidio en adultos mayores colombianos. Revista Cultura del Cuidado. 2016;13(1):70-89.

31. Minayo MCS, Figueiredo AEB, Mangas RMN. Estudo das publicações científicas (2002-2017) sobre ideação suicida, tentativas de suicídio e autonegligência de idosos internados em Instituições de Longa Permanência. Ciênc. saúde colet. 2019;24(4):1393-404.

32. Weber I, Gianolla C, Sotero L. Suicídio e violência estrutural. Revisão sistemática de uma correlação marcada pelo colonialismo. Soc. estado. 2020; 35(1):189-228.

33. Bahia CA, Avanci JQ, Pinto LW, Minayo MCS. Lesão autoprovocada em todos os ciclos da vida: perfil das vítimas em serviços de urgência e emergência de capitais do Brasil. Ciênc. saúde colet. 2017;22(9):2841-50.

34. Santos VC, Anjos KF, Silva JK, Santana JM, Araújo IS. Suicídio na adolescência segundo a raça/cor da pele no Brasil. Revista Brasileira de Saúde Funcional. 2018;6(1):6-10.

35. Sena-Ferreira N, Pessoa VF, Barros RB, Figueiredo AEB, Minayo MCS. Fatores de risco relacionados com suicídio em Palmas (TO), Brasil, 2006-2009, investigados por meio de autópsia psicossocial. Ciênc. saúde colet. 2014;19(1):115-26. 
Contribuições específicas de cada autor:

DAS, JFM: Contribuição substancial no esboço do estudo ou na interpretação dos dados;

DAS, JFM: Participação na redação da versão preliminar;

DAS, JFM: Participação na revisão e aprovação da versão final;

DAS, JFM: Conformidade em ser responsável pela exatidão ou integridade de qualquer parte do estudo.

\section{Financiamento:}

O presente trabalho foi realizado com apoio da Coordenação de Aperfeiçoamento de Pessoal de Nível Superior - Brasil (CAPES).

\section{Autor Correspondente:}

Daniel Augusto da Silva

daniel.augusto@unifesp.br

Editor:

Prof. Dr. Felipe Villela Gomes

Recebido: 10/02/2021

Aprovado: 18/06/2021 